\title{
REVIEW
}

\section{PET/MR: a paradigm shift}

\author{
Florian C. Gaertner, Sebastian Fürst, Markus Schwaiger
}

\author{
Technische Universität München, Klinikum rechts der Isar, Department of Nuclear Medicine, Ismaninger Str. 22, 81675 \\ München, Germany
}

Corresponding address: Dr Florian C. Gaertner, Klinikum rechts der Isar der TU München, Nuklearmedizinische Klinik und Poliklinik, Ismaninger Str. 22, 81675 München, Germany.

Email: florian.gaertner@tum.de

Date accepted for publication 1 November 2012

\begin{abstract}
More than a decade ago, multimodality imaging was introduced into clinical routine with the development of the positron emission tomography (PET)/computed tomography (CT) technique. Since then, PET/CT has been widely accepted in clinical imaging and has emerged as one of the main cancer imaging modalities. With the recent development of combined PET/magnetic resonance (MR) systems for clinical use, a promising new hybrid imaging modality is now becoming increasingly available. The combination of functional information delivered by PET with the morphologic and functional imaging of MR imaging (e.g., diffusion-weighted imaging, dynamic contrastenhanced MR imaging and MR spectroscopy) offers exciting possibilities for clinical applications as well as basic research. However, the differences between CT and MR imaging are fundamental. This also leads to distinct differences between PET/CT and PET/MR not only regarding image interpretation but also concerning data acquisition, data processing and image reconstruction. This article provides an overview of the principal differences between PET/CT and PET/MR in terms of scanner design and technology, attenuation correction, speed, acquisition protocols, radiation exposure and safety aspects. PET/MR is expected to show advantages over PET/CT in clinical applications in which MR is known to be superior to CT due to its high intrinsic soft tissue contrast. However, as of now, only assumptions can be made about the future clinical role of PET/MR, as data about the performance of PET/ MR in the clinical setting are still limited. The possible future clinical use of PET/MR in oncology, neurology and neurooncology, cardiology and imaging of inflammation is discussed.
\end{abstract}

Keywords: Positron emission tomography; magnetic resonance tomography; PET/MR; oncology; neurology; cardiology.

\section{Introduction}

With the development of positron emission tomography (PET)/computed tomography (CT), multimodality imaging entered the clinical arena more than a decade ago ${ }^{[1]}$. The success of the hybrid imaging technique is primarily based on the delivery of complementary information. Mainly morphologic imaging modalities such as CT produce excellent anatomic images, however information on tissue metabolism is limited. PET can deliver this valuable functional information, e.g., about glucose consumption, which is important for oncologic staging, for example, albeit with only sparse anatomic detail and limited spatial resolution. Integration of the two into a single device facilitates exact fusion, thus providing an anatomic reference for the functional information, which is very helpful for exact lesion localization, for example. As a result of this synergy, the information obtained in an examination is richer, leading to more accurate image interpretation. Regarding oncology, which is now the main indication for a PET/CT scan, improvements in staging and restaging accuracies over stand-alone PET or CT have been established for different cancers and, overall, PET/CT has emerged as an important cancer imaging modality ${ }^{[2]}$. Furthermore, CT is utilized as the basis for attenuation correction of the PET data, which supersedes lengthy transmission scans using rotating radioactive sources as in solitary PET imaging. Therefore, the advantages of PET/CT over PET are not only increased confidence in image evaluation and fewer equivocal interpretations but also increased speed and throughput ${ }^{[3]}$. From an economic point of view, 
Table 1 Comparison of PET/CT and PET/MR

\begin{tabular}{|c|c|c|}
\hline & $\mathrm{PET} / \mathrm{CT}$ & $\mathrm{PET} / \mathrm{MR}$ \\
\hline Scanner designs & Sequential: tandem & $\begin{array}{l}\text { Sequential: tandem, shuttle } \\
\text { Simultaneous: insert, fully integrated }\end{array}$ \\
\hline Attenuation correction & $\begin{array}{l}\text { Bilinear transformation of CT data: possible } \\
\text { source of artifacts: metal implants }\end{array}$ & $\begin{array}{l}\text { Atlas-based algorithms: possible sources of artifacts: individual } \\
\text { anatomic variations (e.g., organ configuration, resected } \\
\text { organs, implants) } \\
\text { Segmentation-based algorithms: possible sources of artifacts: metal } \\
\text { implants, bony structures, lungs, edge of transverse MR field of } \\
\text { view }\end{array}$ \\
\hline Speed & Short examination times $(<10 \mathrm{~min})$ & $\begin{array}{l}\text { Longer examination times (depending on scanner design and } \\
\text { MR protocols used) }\end{array}$ \\
\hline & High throughput & Lower throughput \\
\hline Protocols & $\begin{array}{l}\text { Number of protocols manageable: } \\
\text { native low-dose CT; diagnostic } \\
\text { contrast-enhanced CT }\end{array}$ & $\begin{array}{l}\text { Diagnostic MR protocols are more variable } \\
\text { Protocols have to be adapted individually for each patient }\end{array}$ \\
\hline & $\begin{array}{l}\text { Specialized protocols: e.g., multiphase } \\
\text { (abdomen) or head and neck }\end{array}$ & $\begin{array}{l}\text { Time-saving protocols can be laborious to establish } \\
\text { Compromise between number of MR sequences and a reasonable } \\
\text { examination time }\end{array}$ \\
\hline Radiation exposure & $\begin{array}{l}\text { Depending on radiotracer and CT protocol, } \\
\text { CT component may take up to } 70 \% \text { of } \\
\text { total dose in diagnostic CT protocols }\end{array}$ & $\begin{array}{l}\text { Only depending on radiotracer } \\
\text { No additional ionizing radiation by the MR component } \\
\text { (especially relevant for pediatric and nononcologic patients) }\end{array}$ \\
\hline Safety considerations & $\begin{array}{l}\text { Radiation exposure } \\
\text { Contrast-induced nephropathy } \\
\text { Anaphylactoid reactions }\end{array}$ & $\begin{array}{l}\text { Ferromagnetic passive implants (e.g., prostheses, heart valves) } \\
\text { Active implants (e.g., pacemakers, defibrillators) } \\
\text { Nephrogenic systemic fibrosis (NSF) }\end{array}$ \\
\hline $\begin{array}{l}\text { Standardization and } \\
\text { comparability }\end{array}$ & $\begin{array}{l}\text { Highly standardized } \\
\text { Radiodensity comparable over different } \\
\text { models and manufacturers } \\
\text { (Hounsfield units) } \\
\text { Radiodensity proportional to tissue } \\
\text { concentration of contrast agent }\end{array}$ & $\begin{array}{l}\text { Standardization can be challenging } \\
\text { No direct comparability of signal intensity over different sequence } \\
\text { parameters, models, and manufacturers } \\
\text { Signal intensity usually not proportional to concentration of } \\
\text { contrast agent }\end{array}$ \\
\hline Clinical use & $\begin{array}{l}\text { Clinically established } \\
\text { CT has highest sensitivity for small } \\
\text { pulmonary nodules }\end{array}$ & $\begin{array}{l}\text { Up to now, only limited clinical data available } \\
\text { New research possibilities: e.g., combination with fMRI (DWI, } \\
\text { DCE, MRS), motion correction of PET data }\end{array}$ \\
\hline
\end{tabular}

combined hybrid imaging offers advantages. It has been reported recently that a combined contrast-enhanced PET/CT scan is more cost-effective than the two examinations performed separately ${ }^{[4]}$.

Since their introduction into clinical routine in 2001, hybrid PET/CT devices have largely replaced solitary PET scanners in many centers worldwide, which reveals the wide acceptance of hybrid imaging, especially in oncology. By 2006, clinical solitary PET-only scanners were no longer commercially available, and PET/CT has been one of the fastest growing medical imaging modalities $^{[5]}$.

With the recent development of combined PET/magnetic resonance (MR) systems for clinical use, a promising new hybrid imaging modality is now becoming increasingly available. It is well established that MR imaging (MRI) delivers better soft tissue contrast than CT and is thus especially advantageous in body regions with closely adjacent different soft tissue types, including the brain, the abdomen and the pelvis. Next to morphological imaging, MRI also provides functional imaging such as MR spectroscopy, diffusion-weighted imaging (DWI), perfusion imaging and functional MRI (fMRI), e.g., blood oxygen level dependent imaging (BOLD). Combined with the additional functional information delivered by PET imaging, MRI offers exciting new possibilities for clinical applications as well as basic research.

As the differences between CT and MRI are fundamental, so are the differences between PET/CT and PET/ $\mathrm{MR}$, not only regarding image interpretation but also concerning data processing and image reconstruction, the general setup of clinical examination protocols, the contrast agents used, clinical indications and contraindications, examination speed and patient throughput, radiation exposure of the patients and staff, requirements for the medical and technical staff, costs and collaboration between nuclear medicine and radiology (Table 1).

This article outlines the principal differences between PET/CT and PET/MR from the technological and clinical point of view and discusses the possible future use of $\mathrm{PET} / \mathrm{MR}$ in the clinical setting.

\section{Differences between PET/CT and PET/MR}

\section{Scanner design and detector technology}

The concept of a combined PET/CT scanner was developed in the early 1990s. The initial PET/CT scanners took advantage of low-cost PET scanner designs that 


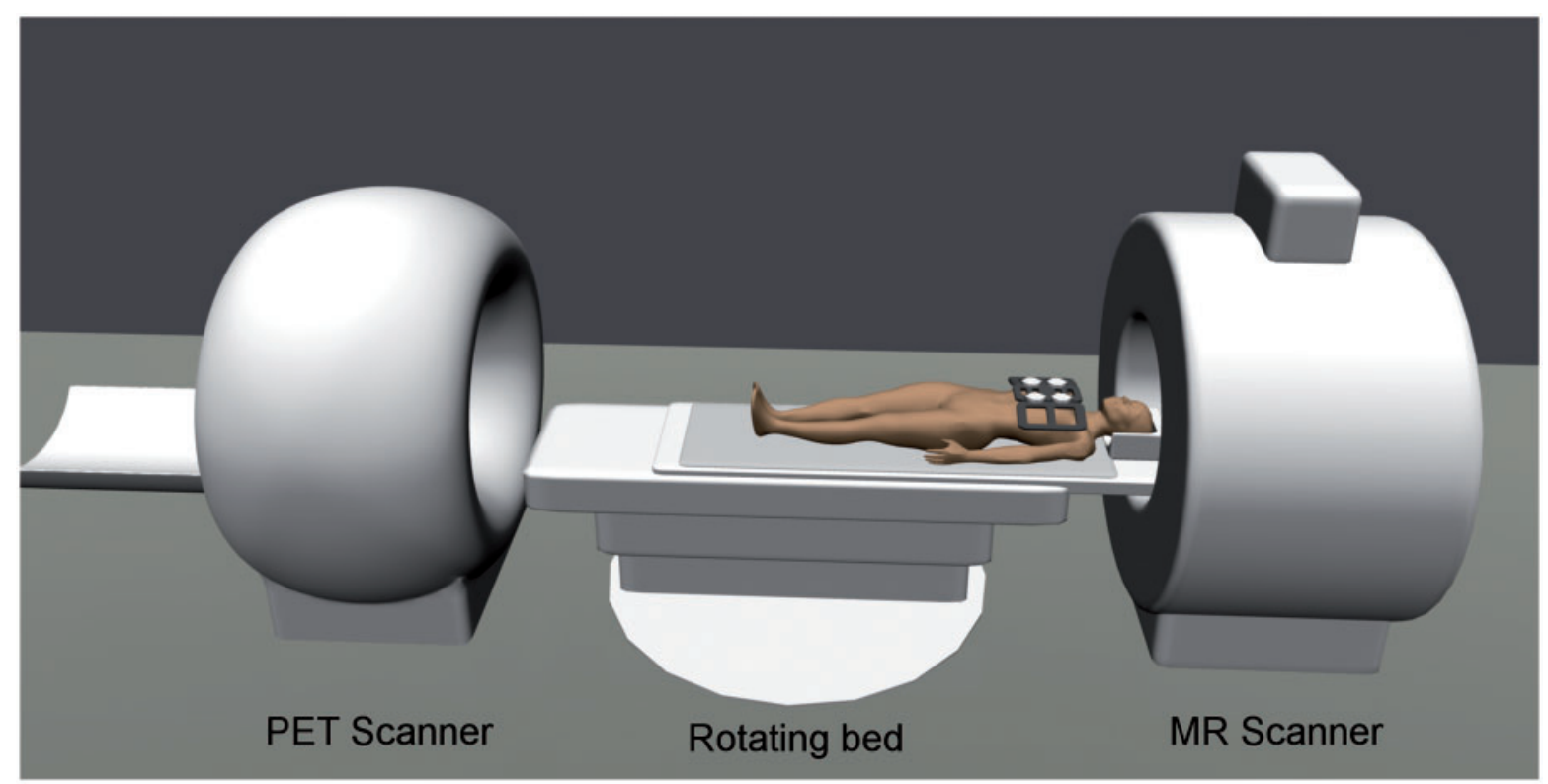

Figure 1 The tandem design is the most straightforward system design for PET/MR scanners. In principle it is the same strategy used in PET/CT scanners, with the 2 modalities physically separated from each other, connected by a common patient bed, on which the patient is moved from one modality to the other sequentially. This approach is advantageous as, aside from shielding, only small technical adjustments are needed regarding the PET and MR devices. However, this approach does not allow simultaneous acquisition of PET and MR data. Moreover, sequential scanning leads to longer examination times for the patient, and one subsystem is always idle. Image is courtesy of Gaspar Delso.

were based on rotating detector blocks rather than on complete detector rings. It was proposed to fill in the gaps between them with the main components required by a CT scanner, an X-ray source and detector. This concept would constitute a fully integrated PET/CT scanner, allowing the truly simultaneous acquisition of PET and CT data. However, it became evident that the space requirements of the $\mathrm{CT}$ components did not allow for such an incorporation, and it was abandoned ${ }^{[5]}$. The PET/CT design that first became reality as a prototype in 1998 was a tandem design that consisted of a coupled 1-slice spiral CT tomograph and a rotating bismuth germanate (BGO) PET detector block in one single gantry. PET and CT data were acquired sequentially by moving the patient bed from one modality to the other. Although current system designs reflect technological advances in both modalities (e.g., multislice spiral CT, full-ring PET detectors and advanced scintillation materials), the different models currently offered by the major medical companies are still based on the tandem design.

The tandem or sequential configuration with two physically separated subsystems also constitutes the most straightforward design for PET/MR systems (Fig. 1). This approach is least demanding from a technical point of view, as it does not require fundamental changes to already established PET and MR technologies. Despite the physical separation, it takes more than just the installation of two devices in the same room and the creation of a logical link between them. Additional care has to be taken to adequately shield the PET detectors, especially the commonly used photomultiplier tubes (PMTs), which are affected by low magnetic fields and cease to work in high magnetic field strengths ${ }^{[6]}$. Shielding has to be applied to minimize disturbances of the magnetic field of the MR scanner and prevent mutual interference of the MR gradient fields and radiofrequency (RF) pulses on the one hand and the front-end electronics of the PET unit on the other. Shuttle solutions, which allow the installation of the PET and MR scanners in separate rooms, avoid these issues. A patient bed with an immobilization device is used to transfer the patient from one modality to the other, minimizing patient motion during transport. The main disadvantage of sequential PET/MR scanner designs is the obvious lack of simultaneous acquisition, resulting in relatively long total examination times. Furthermore, in designs with both devices located in the same room and one common patient bed, one of the modalities is always idle. Therefore, shuttle systems are advantageous from an economic point of view, as the first device can be used for the next patient as soon as the previous patient is moved to the second device ${ }^{[7]}$.

PET inserts do allow simultaneous PET and MR acquisition. This not only shortens total imaging time for the patient and reduces possible misregistration artifacts due to patient motion between the two different scans but also opens up opportunities for new applications. In this approach, an MR-compatible PET detector ring is placed inside an existing MR scanner (Fig. 2). This does not require any changes to the MR device. Due to their proximity, however, the PET detectors have to be 


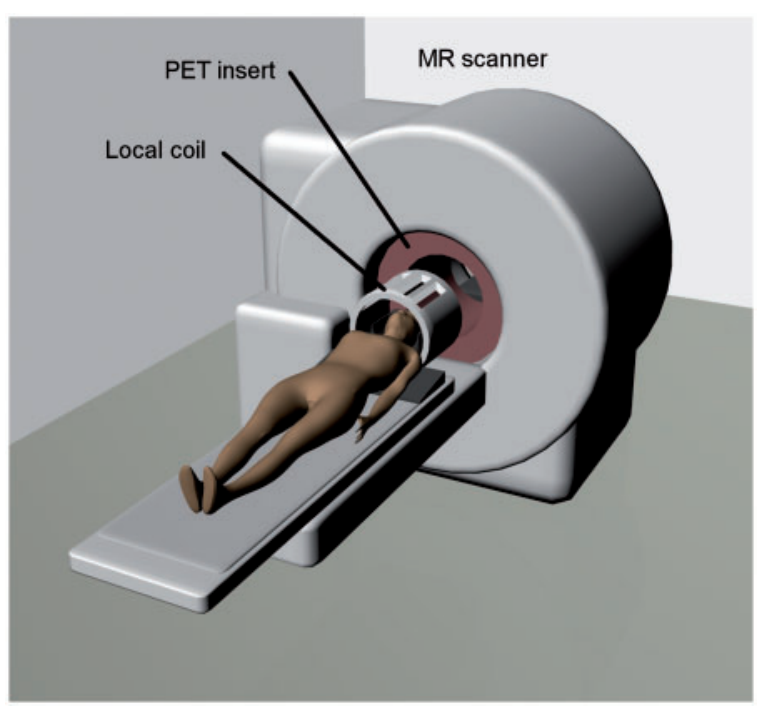

Figure 2 The insert architecture consists of an MR-compatible PET detector ring, which is introduced into an existing MR scanner, allowing truly simultaneous acquisition of PET and MR data. This approach demands a complete redesign of the PET detector technology, as the conventionally used PMTs cease to work in the high magnetic field strengths of the MR scanner. As only minimal changes to preexisting MR devices are needed, this approach would constitute a cost-effective way for institutions to gain access to PET/MR imaging. However, the PET insert substantially narrows the MR gantry, which limits this approach to preclinical imaging and to imaging of the head and extremities in patients. Image is courtesy of Gaspar Delso.

redesigned completely to minimize the mutual disturbance and interference already mentioned. PMTs have to be replaced altogether or, as was the case in the first MR-compatible PET detector designs, positioned several meters away from the MR scanner, where the magnetic field strength is low enough. Optical fibers were then used to guide the light pulses emitted by the scintillator crystals to the photodetectors ${ }^{[8]}$. Avalanche photodiodes (APDs), on the other hand, are insensitive to magnetic fields and can therefore be used to detect scintillation light directly in the bore of the MR scanner ${ }^{[9]}$. Furthermore, APDs are very compact compared with PMTs, which is advantageous regarding the limited space inside the gantry of an MR scanner. Drawbacks of APD detectors include low gain and lower energy and timing resolution compared with PMTs. Furthermore, APDs require very stringent temperature adjustment ${ }^{[6]}$. In practice, the insert design is very useful for preclinical small-animal imaging and clinical imaging of the brain and extremities, but not feasible for clinical whole-body imaging, as the gantry diameter is substantially narrowed. It constitutes a cost-effective way for institutions to gain access to PET/MR imaging, still allowing conventional MR examinations as well ${ }^{[6]}$.
Fully integrated whole-body PET/MR is a recent development that allows truly simultaneous imaging of patients (Fig. 3). This approach is technologically the most challenging and most expensive as it requires major modifications of both the PET and MR technology. Fully integrated PET/MR scanners are now commercially available and the first scanners were installed in clinical centers in 2010. The design of the hybrid Siemens PET/MR scanner (Biograph mMR) is mainly based on a Siemens Verio 3 Tesla MR device, into which PET detector rings using lutetium orthosilicate (LSO) crystals and water-cooled APD photodetectors have been added between the gradient and body coils. This results in a smaller bore of the Biograph mMR $(60 \mathrm{~cm})$ compared with the Verio MR scanner $(70 \mathrm{~cm})$. Detailed performance characteristics of this scanner and data about the feasibility of PET/MR in comparison with $\mathrm{PET} / \mathrm{CT}$ in oncology patients have been published by our group previously ${ }^{[10,11]}$. Its clinical performance is currently being evaluated in trials.

\section{Attenuation correction}

A prerequisite for quantitative PET image information is the correction of emission data for attenuation. In PET/ CT this is achieved with the help of the CT component. As it delivers direct information about the gamma ray attenuation properties of tissue, the generation of attenuation maps containing attenuation coefficients for $511 \mathrm{keV}$ electromagnetic radiation from the CT data by bilinear transformation is quite straightforward ${ }^{[12]}$. However, errors and artifacts may occur in the region of metal implants. On the other hand, the MR signal depends on the density of protons and the relaxation properties of their spins in tissue, but does not deliver information about the absorption of ionizing radiation. Thus, the generation of attenuation maps with PET/MR is more complex.

Different approaches have been investigated concerning attenuation correction of PET/MR data, which can be assigned to 2 categories: atlas-based methods and segmentation-based approaches ${ }^{[13]}$. Atlas-based methods typically use a set of standard population-based MR images and corresponding predefined attenuation maps. Basically, the standard MR image is coregistered to the MR data for the individual patient, and the same spatial transformation can then be applied to the corresponding predefined attenuation map to generate a map matching that patient. The advantage of atlas-based methods is the possibility of easily including bony structures in the attenuation maps. However, individual anatomic anomalies cannot be accounted for, e.g., postoperative states (resected organs, implants) or different organ configurations (e.g., filling state of the stomach or urinary bladder). Segmentation-based algorithms build on the image information of the acquired MR data for tissue classification. Voxels are assigned (segmented) to tissue types, such as air, water, and fat, based on their MR signal 


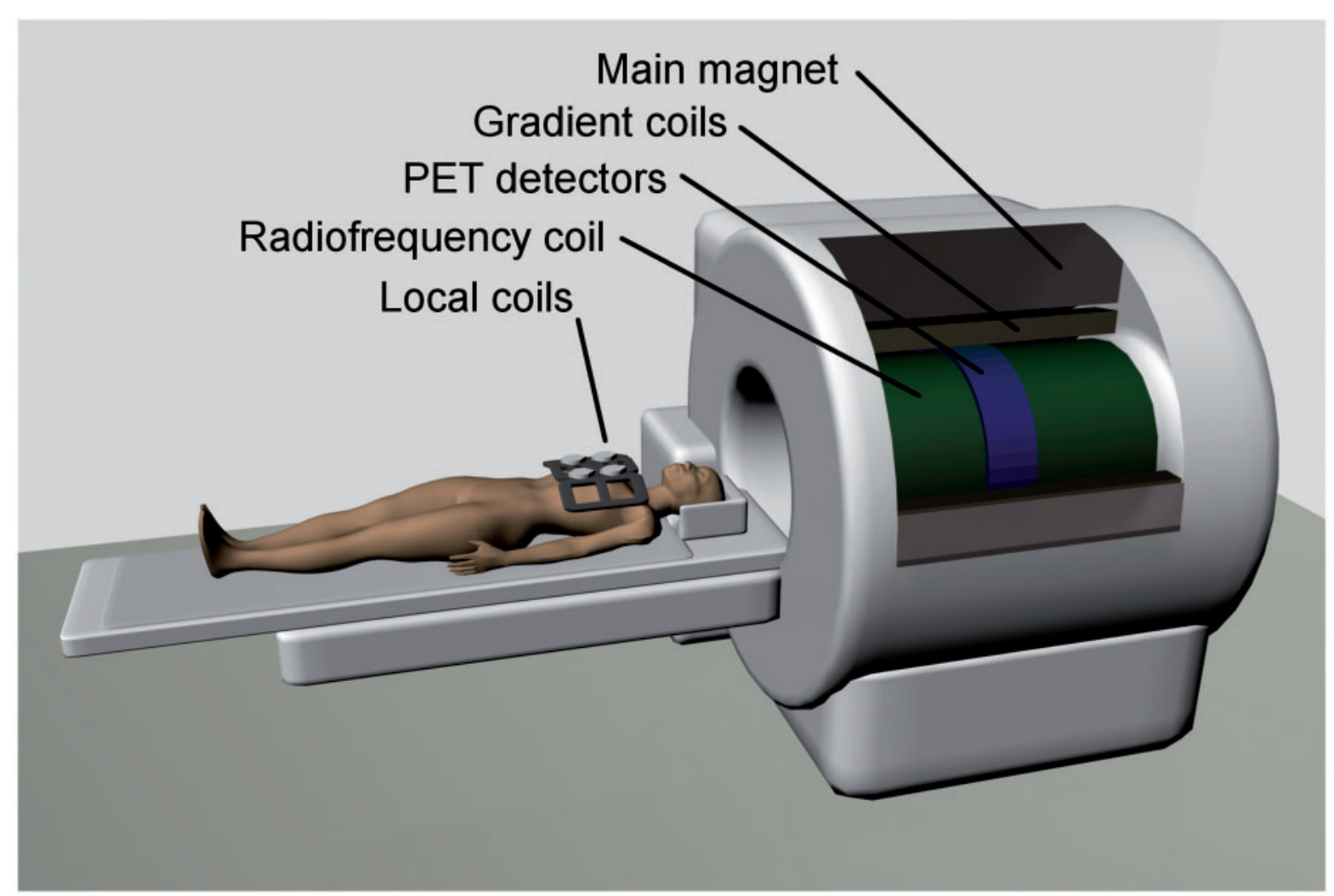

Figure 3 Fully integrated PET/MR designs for clinical whole-body imaging have recently become commercially available. These scanners allow truly simultaneous acquisition of PET and MR data in patients, which reduces the examination time in comparison with sequential approaches, and opens up new possibilities for clinical research. This approach is most challenging from the technological point of view, as it demands a complete redesign of the PET detector ring, as well as substantial changes to the MR technology. PMTs have been replaced by APDs, which allow detection of the scintillation light even in high magnetic field strengths, and the PET detector ring has been integrated between the RF and gradient coils of the MR gantry. Image is courtesy of Gaspar Delso.

properties. One way to accomplish this is the acquisition of images with a 2-point Dixon sequence, which delivers water- and fat-weighted images ${ }^{[14]}$. The technical challenges regarding segmentation-based approaches might be most evident in the separation of cortical bone and air. These show the largest differences in absorption coefficients, but the proton density and thus MR signal intensities of both are typically low. Some algorithms simply ignore bone in the attenuation map generation. Although its attenuation coefficient is much higher than that of soft tissue, the impact of neglecting bones on attenuation correction has been reported to be low in clinical practice ${ }^{[15]}$, except in the head and in lesions near bone structures. The incorporation of ultrashort echo time (UTE) sequences, which generate an MR signal even from cortical bone might be a solution to this prob${ }^{l e m}{ }^{[16]}$. As with CT, artifacts may also arise in the case of metal implants. These cause a loss of MR signal and thus result in misclassification of surrounding voxels as air. MR surface coils might not be included in the attenuation correction and therefore cause additional artifacts. Further artifacts may occur if the lung compartment is wrongly classified as air, or due to distortion and signal loss at the edge of the transverse field of view of the MR image (Fig. 4). Efforts are made to recover the patient surface in these areas by additionally incorporating atlasbased data or by image processing of the non-attenuation correction PET data to subsequently correct the attenuation correction map. Depending on the segmentation method, administration of gadolinium-based MR contrast agents might also adversely affect the generation of attenuation maps.

\section{Speed}

Technological advances in the last 15 years have led to significant gains in speed of PET acquisitions, among them the development of new scintillation materials, such as LSO. The most significant increase in speed, however, was achieved by the introduction of PET/CT and the use of CT instead of transmission data for attenuation correction. This reduced the total examination time by up to $40 \%{ }^{[17]}$. With modern multislice CT scanners, high-quality whole-body examinations can be performed in just a few seconds. On state-of-the-art PET/ CT scanners, a whole-body examination can be 




Figure 4 (A) Attenuation map of one PET bed position generated from the 2-point MR Dixon sequence using segmentation algorithms as implemented on the Biograph mMR without obvious artifacts. (B) Metal, such as in hip implants, does not yield an MR signal and reduces the signal in its vicinity (arrows). (C) The resulting attenuation map as generated by the Biograph mMR therefore wrongly exhibits air instead (black arrows). Furthermore, truncation artifacts may occur at the edge of the transverse field of view of the MR (white arrows). (D) A transaxial slice of the fusion of an attenuation map with the emission image clearly shows the truncation of arms in the attenuation map due to the smaller field of view of the MR (arrows).

completed in less than $10 \mathrm{~min}$, including fully diagnostic CT protocols. Short examination times are not only advantageous regarding economics and patient throughput, but also improve patient comfort and help to reduce misalignment artifacts due to patient motion during the examination.

The speed of PET/MR examinations is strongly dependent on the scanner design and MR protocols. The sequential acquisition of PET and MR data in scanners based on the tandem design have the longest total examination time for the patient.

Scanner designs allowing simultaneous PET and MR acquisitions (insert systems and fully integrated systems) may help to keep scan times shorter for the patient.
However, in our clinical experience, we observed that with a fully integrated PET/MR scanner, total examination times tend to be longer compared with PET/CT. The technician's task of planning a PET/MR acquisition is more complex and thus time consuming. Patient preparation, positioning on the scanner, and unloading significantly contribute to the patient room time and are prolonged by, e.g., applying and connecting the MR coils. Additional diagnostic MR sequences are another major contribution to examination times, e.g., within organ-specific protocols and sequences after the application of intravenous contrast agents that are not acquired before the completion of whole-body PET scans. Not only examination times tend to be longer for PET/MR 
but also image interpretation and reporting take longer due to their complexity.

\section{Acquisition protocols}

Examination protocols for PET/MR tend to be diverse, strongly depend on the indication, and are complex and laborious to design, especially simultaneous PET/MR protocols. Time-saving protocols are desirable, achieving the right compromise between the MR sequences needed and a reasonable examination time and then efficiently intercalating the PET scans and MR sequences.

The most basic PET/MR protocol consists of MR localizers to allow planning of the PET bed positions, then performing the PET emission scans while simultaneously acquiring MR sequences for attenuation correction, e.g., a 2-point Dixon sequence for each bed position. The resulting Dixon images (in-phase, opposed-phase, waterweighted and fat-weighted) can also be used for simple anatomic correlation of PET lesions, analogous to a lowdose CT scan ${ }^{[18]}$. Examples of PET/MR protocols using additional diagnostic MR sequences are given in Fig. 5.

\section{Radiation exposure and safety aspects}

MRI is based on static and rapidly alternating magnetic fields with high field strengths and radiofrequency (RF) electromagnetic fields ${ }^{[19]}$, but does not utilize ionizing radiation. Therefore, performing a PET/MR examination instead of a PET/CT examination reduces the exposure of ionizing radiation for the patient. This is of special relevance for pediatric and nononcologic indications. However, PET/MR may be associated with a higher radiation exposure for the technicians performing the examination, as patient positioning takes considerably longer, particularly when attaching and connecting the MR surface coils, during which the technician is in close proximity to the gamma-emitting patient.

There is little evidence that static magnetic fields, even at high field strengths, have a biological effect on cells or tissues, although sensations of nausea, vertigo and metallic taste have been reported at field strengths above $2 \mathrm{~T}^{[20]}$. The rapid switching of the magnetic gradient fields induces currents in the patient's body, which may stimulate nerve and muscle cells. The RF pulses used for excitation of the spin system lead to a temperature increase in the tissue. During the examination, the core temperature of the patient should not increase more than $0.5^{\circ} \mathrm{C}$ and $1^{\circ} \mathrm{C}$ in normal and controlled operating modes, respectively, which is estimated by the specific absorption rate (SAR) during the examination. However, there is a different sort of safety risk in MRI. Any ferromagnetic object is accelerated in a magnetic field and can therefore cause fatal harm to the patient, staff, and equipment. Objects with ferromagnetic properties can include passive and active implants (e.g., aneurysm clips, wires, mechanical heart valve prostheses and pacemakers, defibrillators, cochlear implants, medication pumps, etc.), foreign bodies in the patient (e.g., posttraumatic metallic foreign bodies, bullets, etc.) and other objects that are inadvertently brought into the examination room (e.g., patient bed, oxygen flasks, etc.). These may pose absolute contraindications for a PET/MR examination and careful interview of the patient and investigation about the MR compatibility of implants is required before the examination.

Safety risks may also arise from contrast agents used for diagnostic imaging. Anaphylactoid reactions are very rare in MR examinations, and thus, in this regard, Gdbased contrast agents are considered safer than CT contrast media. However, it was recognized a few years ago that MR contrast media are associated with nephrogenic systemic fibrosis (NSF), a rare but potentially life-threatening complication that leads to rapid fibrosis of the skin and internal organs, including the heart, liver, lungs, and kidneys. Presumably the release of small amounts of free gadolinium from the $\mathrm{Gd}$ complexes is the reason for the development of NSF, and preexisting severe renal impairment poses a risk factor for the development of NSF ${ }^{[21]}$. Cyclic Gd-chelates, e.g., Gd-DOTA (tetraazacyclododecane-tetraacetic acid), are more stable than linear complexes such as, e.g., Gd-DTPA (diethylene triamine pentaacetic acid) and clinical data indicate that the risk of developing NSF after the application of cyclic Gd-chelates is significantly lower, thus cyclic complexes are preferred in patients with preexisting severe renal impairment ${ }^{[22]}$.

\section{Standardization}

The radiation emitted by a CT scanner is not monochromatic, but instead a spectrum of x-rays. This spectrum depends on the scanner hardware and might not only be different for scanners from different manufacturers but also for different models by the same manufacturer. Because the absorption coefficient of any matter is a nonlinear function of energy, this means that the CT values of the same object obtained on different scanners are not necessarily equal. Standardization, however, is straightforward.

Values in reconstructed CT images are conventionally given in Hounsfield units (HU). The Hounsfield scale ideally converts the linear absorption coefficients of materials such that air is assigned $-1000 \mathrm{HU}$ and water $0 \mathrm{HU}$. By calibrating a CT scanner using air and water, any HU measured on that scanner can be corrected and the ideal HU calculated ${ }^{[23]}$. These ideal HUs facilitate standardization of CT examinations over different scanner models, manufacturers, and institutions. Furthermore, it allows the direct and quantitative measurement of the concentration of radiodense contrast agent in the tissue.

The MR signal is affected by many more factors. Sequences are designed to give more weight to certain tissue constituents, such as fat or water. Manufacturers provide their own sets of hardware-specific and often 

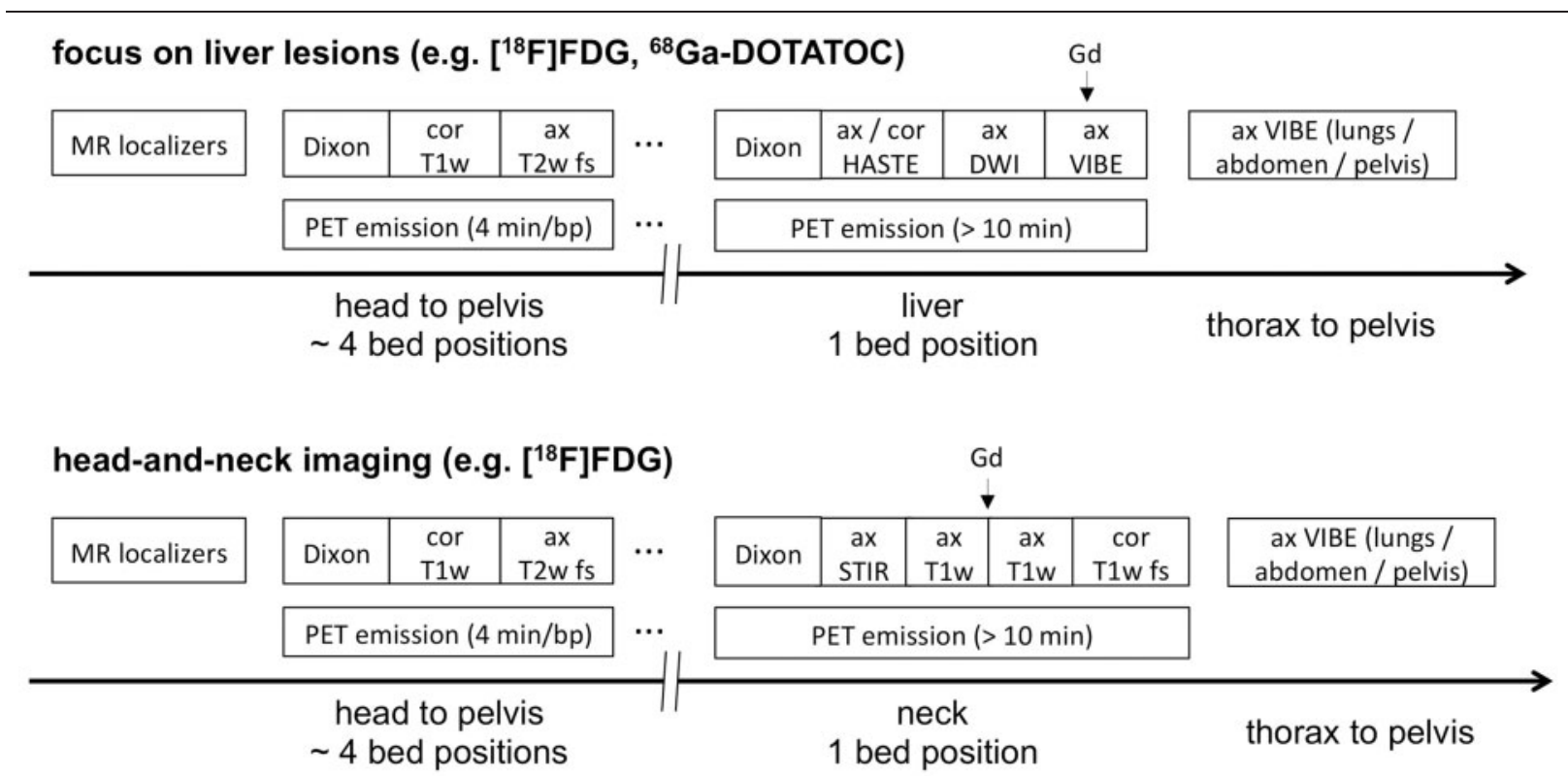

\section{prostate cancer (e.g. $\left[{ }^{11} \mathrm{C}\right]$ choline)}

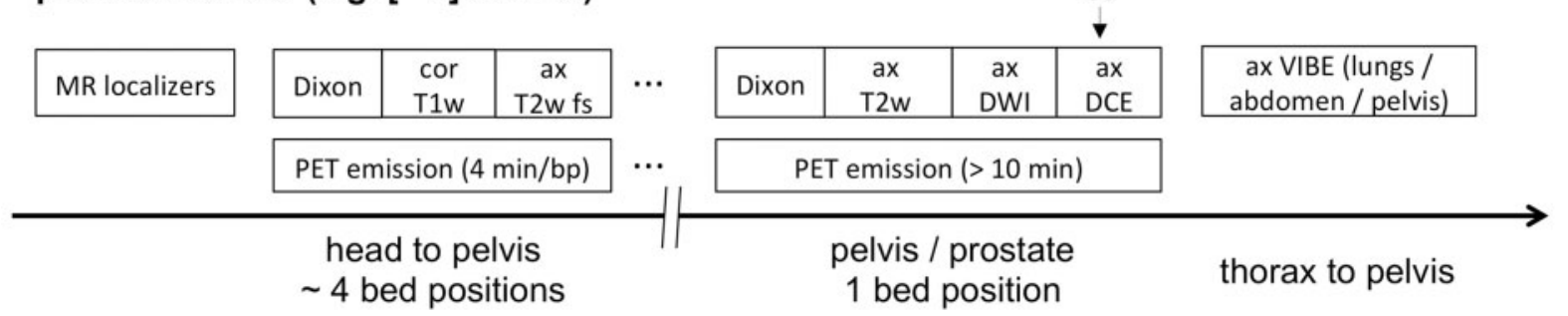

Figure 5 Sample workflows for oncologic diagnostic PET/MR protocols. First, MR localizers are performed for planning of PET bed positions. Then, PET emission data are acquired. Here, the body trunk can be covered by about 4 bed positions (e.g., 4 min emission time per bed position). Simultaneously, a Dixon sequence for attenuation correction and additional diagnostic MR sequences are run at each bed position, e.g., a coronal T1 turbo spin echo (TSE) and an axial T2 half Fourier acquisition single shot turbo spin echo (HASTE) with fat suppression. However, as the application of MR contrast agent may affect the generation of the attenuation correction map, Gd should not be administered before all MR Dixon sequences are completed. Optional diagnostic MR sequences are then performed over the region of special interest depending on the clinical indication. Contrast agents can be injected if necessary. During this phase, additional PET data may be collected at the corresponding bed position using long emission times, delivering potentially lower-noise PET images. Concluding MR scans may be acquired covering the body trunk (thorax, abdomen, pelvis) after the application of Gd, e.g., axial T1 volumetric interpolated breath-hold examination (VIBE) sequences. (A) Sample workflow with focus on the liver using e.g., $\left[{ }^{18} \mathrm{~F}\right]$ FDG or ${ }^{68}$ Ga-labeled somatostatin analogues for staging of patients with neuroendocrine tumors. (B) Sample workflow with focus on the neck for staging of patients with head and neck cancer. (C) Sample workflow for imaging of patients with prostate cancer using choline PET/MR. Image is adapted from Ref. ${ }^{[55]}$.

unique sequences. Adjusting the acquisition parameters of a sequence can also alter contrast. In general, the signal increases with increasing field strength, but not all sequences are suitable for all field strengths. Furthermore, the MR signal intensity usually is not proportional to the concentration of Gd-based contrast agent in the tissue. Dedicated coils are used to detect the MR signal during an acquisition, the design and position of which influence the received signal as well. The nearer these coils can be placed to the body region or the object under investigation, the higher the signal-to-noise ratio and the overall signal strength will be. Dedicated protocols exist for almost any indication, whereas their exact composition and sequence parameters are determined by the operator. Such dependency on hardware, acquisition, and operator make quantification and standardization of MR data extremely difficult at the present time.

\section{Research possibilities of PET/MR}

Hybrid PET/MR is an ideal platform for basic biomedical and clinical research. One advantage is the inherent 
coregistration of the two modalities. A stimulating further possibility of fully integrated PET/MR devices is the truly simultaneous acquisition of PET and MR data. This could be used for motion correction and gating of PET data without external sensors by incorporation of MR information into the PET list mode data stream, allowing postprocessing of PET data. Another exciting base for research applications is the possibility of simultaneously acquiring dynamic PET data and fMRI sequences. This would allow the examination of organs and tissues under the same physiologic conditions by the two different modalities.

Neurologic research might gain most from truly simultaneous PET/MR imaging, as the physiologic state of the brain, e.g., secretion of neurotransmitters, receptor binding, drug-receptor interaction, and cortical activation, changes rapidly. One example would be the simultaneous temporal and spatial assessment of specific ligand-receptor displacement by dynamic PET (e.g., using a radiolabeled dopamine-receptor ligand) and cortical activation by BOLD MRI, while the patient is performing tasks ${ }^{[24]}$.

An example for an oncologic field of research that might profit from simultaneous PET/MR acquisition is tumor perfusion and hypoxia. Tumor hypoxia is known to be a predictive factor for clinical outcome in several tumor entities, especially regarding squamous cell carcinoma of the head and neck. It is largely accepted that tumor hypoxia is not static, but rather a cycling process with fluctuating areas of transient hypoxia in the tumor due to variances in perfusion, which might change in the time frame of just a few minutes ${ }^{[25]}$. The possibility of validating perfusion measured by $\mathrm{PET}$ (e.g., $\left[{ }^{15} \mathrm{O}^{1} \mathrm{H}_{2} \mathrm{O}\right.$ or $\left[{ }^{13} \mathrm{~N} \mathrm{NH}_{3}\right.$ ) with markers of MR perfusion (dynamic contrast-enhanced (DCE)-MRI or arterial spin labeling) under the same physiologic state is exciting. The correlation of simultaneously acquired fMRI (e.g., BOLD MRI, which delivers a measure of the amount of deoxyhemoglobin present in the tissue) and hypoxia-specific PET tracers (e.g., $\left[{ }^{18} \mathrm{~F}\right]$ fluoromisonidazole (FMISO), $\left[{ }^{18} \mathrm{~F}\right]$ fluoroazomycin arabinoside (FAZA) or $\left[{ }^{64} \mathrm{Cu}\right]$-diacetyl-bis-methylthiosemicarbazone (ATSM)) might provide further insights into the complex physiologic processes associated with tumor hypoxia.

\section{Possible clinical advantages of PET/MR}

PET/MR is a young technology and has only recently become available for clinical use. Published data on the clinical performance of PET/MR is still very limited and only assumptions can be made concerning the benefits and future applications of PET/MR in the clinical routine setting.

\section{Oncology}

In oncology, PET/MR might show improvements compared with $\mathrm{PET} / \mathrm{CT}$ in terms of local tumor evaluation.
The assessment of exact tumor localization, size, and potential infiltration of neighboring organs, vessels, and neural structures requires imaging data with high spatial resolution. In many tumor entities, the resulting information is also important to determine the required therapy approach, e.g., the necessary extent of tumor resection, and for radiation therapy planning (Fig. 6). However, the spatial resolution of about $4 \mathrm{~mm}$ in current state-of-the-art clinical PET is limited. Therefore, T-staging primarily relies on the information delivered by high-resolution morphological imaging. PET/MR will probably show advantages in body regions and tumor entities where MRI, due to its high intrinsic soft tissue contrast, is known to be superior to CT (e.g., soft tissue sarcomas, head and neck cancer, breast cancer, and tumors of the parenchymal abdominal organs) ${ }^{[26]}$.

Regarding breast cancer, MR mammography is a highly sensitive tool for preoperative assessment of tumor size and ductal invasion ${ }^{[27]}$. On the other hand, fluorodeoxyglucose (FDG)-PET seems to be advantageous in differentiating unifocal from multicentric lesions, which also influences the therapeutic strategy concerning breast ablation or breast-preserving surgery $^{[28]}$. Combined PET/MR could integrate these advantages into one single examination, while at the same time performing whole-body staging for loco-regional and distant metastases. However, this approach might require specialized positioning devices for breast imaging ${ }^{[26]}$.

Choline PET/CT is a promising modality for restaging of patients with biochemical recurrence of prostate cancer, and first results on its use for therapy response assessment are also promising ${ }^{[29]}$. PET/MR could combine the high sensitivity of choline PET with the high resolution and high soft tissue contrast imaging of MRI and potentially fMRI data such as DWI, DCE-MRI and MR spectroscopy (Fig. 7). Hybrid imaging using choline PET/MR might improve differentiation of tumor recurrence from scar tissue, which can still cause problems in $\mathrm{MRI}^{[30]}$.

For the evaluation of lymph node metastases, CT primarily relies on the size and shape of lymph nodes to differentiate between benign and malignant lesions. The additional metabolic information delivered by FDG-PET/ CT has been shown to significantly improve the diagnostic accuracy of lymph node staging compared with CT alone $^{[31]}$. As whole-body MRI based on T1- and T2weighted images and postcontrast sequences also primarily relies on lymph node size and shape, the advantage of PET/MR over PET/CT for N-staging is debatable. DWI might allow the detection of malignant lymph nodes with higher sensitivity and specificity, although reports in the literature are diverging ${ }^{[32,33]}$. The additional use of ultrasmall iron oxide particles (USPIO) as an MR contrast agent specific for the reticuloendothelial system poses an interesting opportunity for PET/MR to more accurately differentiate reactive tracer uptake in inflammatory lymph nodes from lymph node metastases ${ }^{[34]}$. 


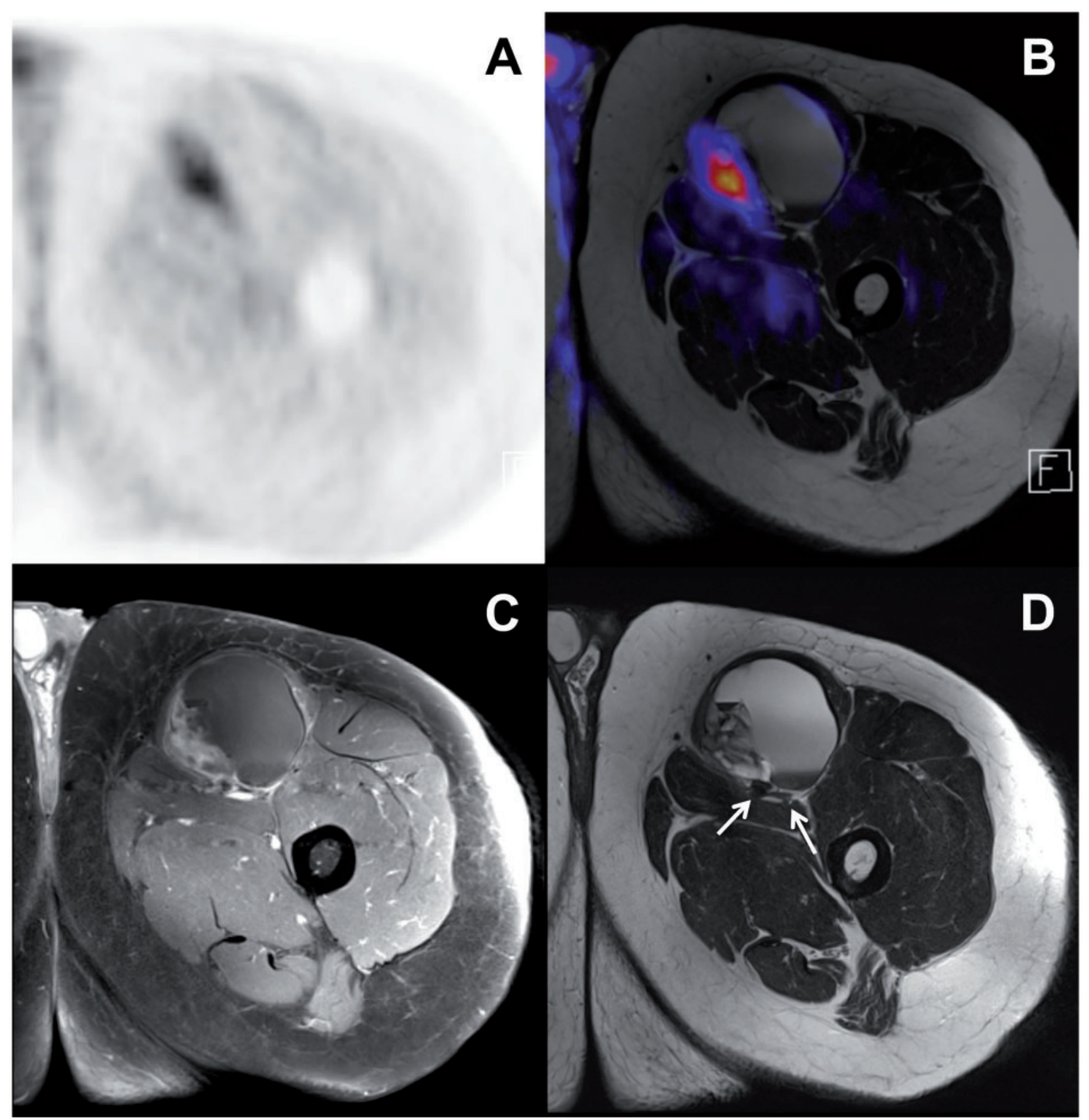

Figure 6 A 58-year-old man with biopsy proven synovial sarcoma of the left thigh. The patient was restaged by PET/MR after neoadjuvant radiotherapy. (A-C) Remaining pathologically increased glucose metabolism (maximum standardized uptake value 7.2) is observed especially in the solid medial tumor part (A, PET; B, PET/MR fusion), which also shows increased contrast enhancement in the fat-suppressed T1-weighted image after the application of Gd (C), consistent with remaining vital tumor tissue after radiotherapy. (D) High-resolution anatomic MR imaging (T2-weighted image) delivers valuable information for planned tumor resection, e.g., the close proximity of the tumor to the femoral vessels and nerve (arrows).

However, USPIO particles have not received regulatory approval in the United States or in Europe ${ }^{[30]}$.

Because MRI is well suited for the detection of cellular infiltration of the bone marrow and whole-body MRI has been reported to be of high diagnostic accuracy for the evaluation of bone metastases ${ }^{[35]}$, PET/MR may be expected to show advantages with respect to the detection of osseous metastatic disease. Moreover, increased physiologic background activity and image degradation caused by respiratory motion decrease the sensitivity of FDG-PET for small liver lesions. On the other hand, MRI using DWI is highly sensitive for the detection of liver metastases $^{[36]}$, making PET/MR a promising combination for their evaluation (Fig. 8). Theoretically, the MR information acquired simultaneously with the PET emission data could be used for motion correction of the PET 


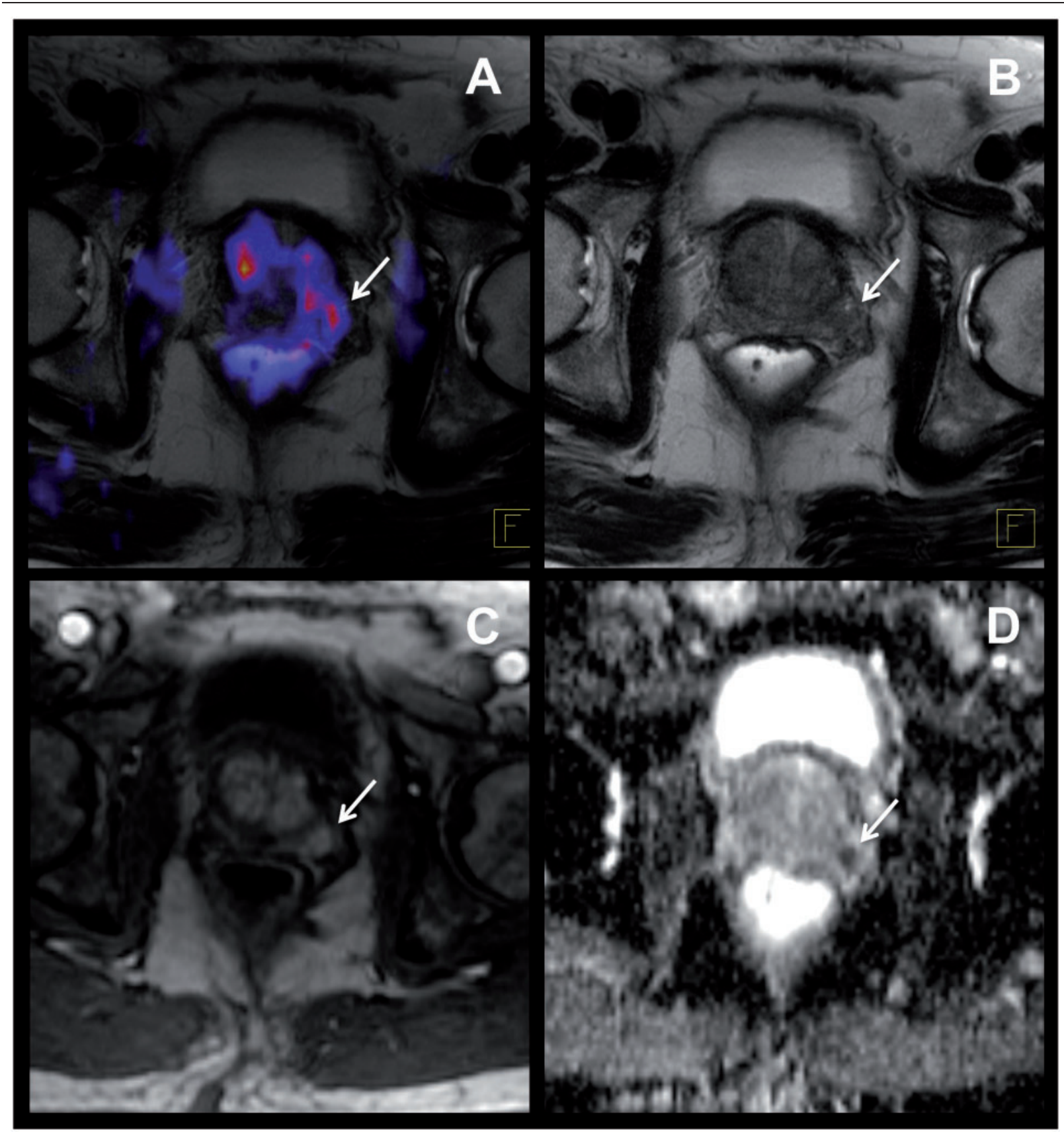

Figure 7 A 69-year-old patient with suspected prostate cancer (prostate-specific antigen $12 \mathrm{ng} / \mathrm{ml}$ ). (A) $\left[{ }^{11} \mathrm{C}\right]$ Choline PET/MR shows pathologically increased tracer uptake in the left peripheral zone of the prostate, suspicious of the primary tumor (arrow). (B-D) Consistent with choline PET, the T2-weighted MR images show a suspicious signal loss in the left peripheral zone (B, arrow), corresponding to an area of increased perfusion in the DCE MR sequence (C, arrow) and a low apparent diffusion coefficient in the diffusion-weighted MR images (D, arrow). Subsequent prostatectomy confirmed a carcinoma of the prostate in the left peripheral zone.

images, improving their diagnostic quality and quantitative information ${ }^{[37]}$, which could prove to be useful not only for evaluation of the liver, but for all organs affected by respiratory motion.

Furthermore, as MRI is the modality of choice for the evaluation of brain metastases, inclusion of diagnostic MR sequences of the brain into whole-body FDG-PET/ MR staging protocols would enable the additional evaluation of cerebral metastases in a convenient onestop-shop examination.

Overall, when identifying the indication correctly, PET/MR may provide a more accurate staging than whole-body PET/CT or whole-body $\mathrm{MRI}^{[26]}$. However, it is well known that the sensitivity of MRI for the detection of small pulmonary nodules $(<3 \mathrm{~mm})$ is limited, and the sensitivity of FDG-PET for small pulmonary nodules 

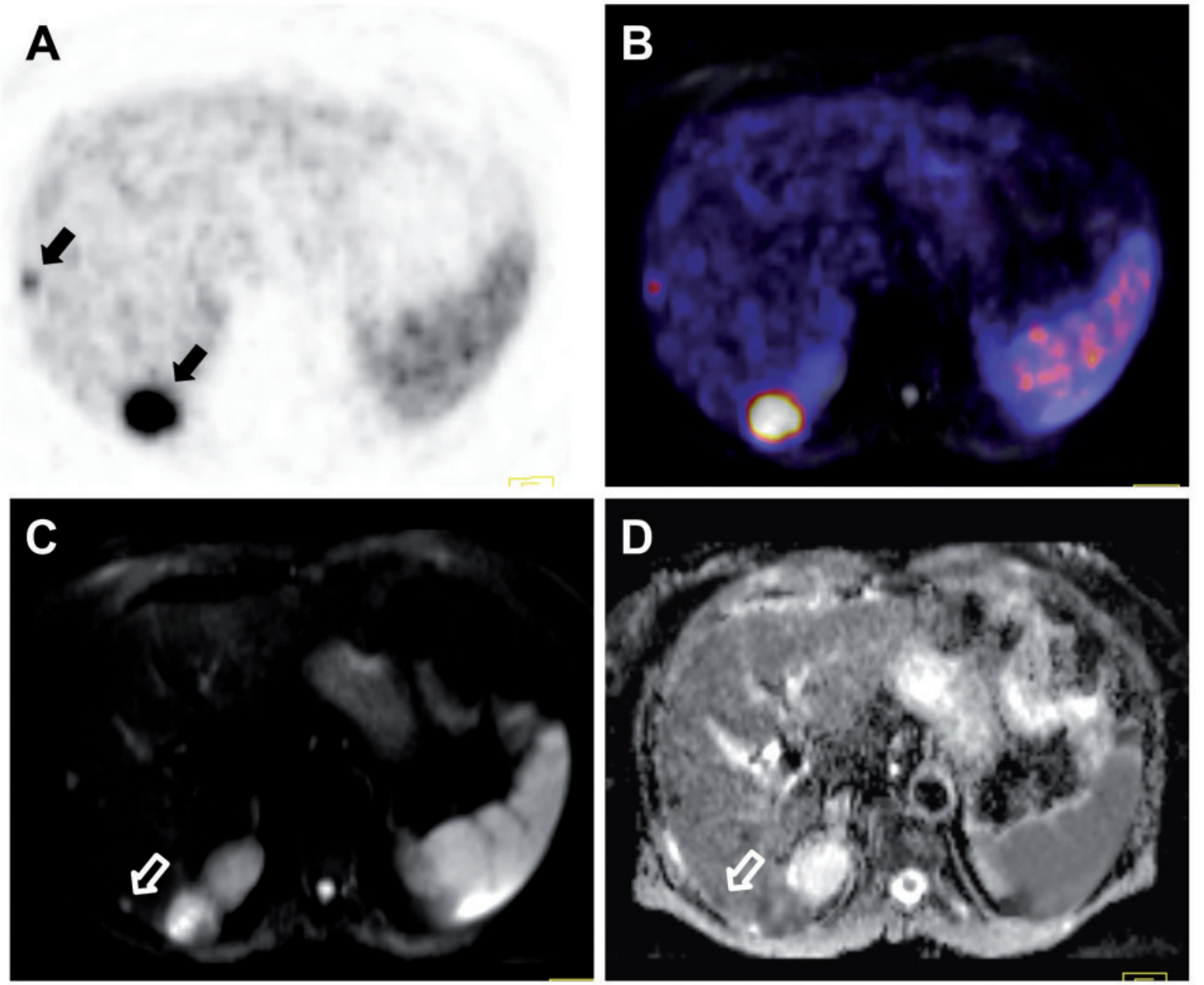

Figure 8 A 73-year-old patient with a neuroendocrine carcinoma of the small intestine and metastatic spread to the liver. ${ }^{68}$ Ga-DOTATOC PET/MR shows increased uptake in 2 of the hepatic lesions, corresponding to overexpression of the somatostatin receptor (black arrows; A, PET; B, PET/MR fusion). (C) Diffusion-weighted MRI shows a third suspicious hepatic lesion that was not detected by PET, possibly due to loss of receptor expression (white open arrow). (D) A low apparent diffusion coefficient of this lesion, however, indicates that it indeed is a metastasis (white open arrow).

is low. As CT has the highest sensitivity for detection of small pulmonary metastases, the indication for a PET/ MR or a PET/CT examination has to be evaluated with care, and a CT of the thorax complementing a wholebody PET/MR will have to be considered in some indications.

\section{Neurology and neurooncology}

PET and MR imaging is an ideal combination for tackling neurologic and neurooncologic clinical problems. MRI is undoubtedly the clinical standard imaging modality for brain tumors, delivering information about tumor localization and size as well as secondary effects such as focal edema or bleeding. Amino acid PET (e.g., $\left[{ }^{18} \mathrm{~F}\right]$ fluoro-ethyl-L-tyrosine) or L- $\left[\right.$ methyl $\left.-{ }^{11} \mathrm{C}\right]$ methionine) can add substantial clinical information to MRI regarding differentiation of tumor tissue from reactive or inflammatory changes and tissue edema, and may help to better delineate the true extent of the tumor tissue, which can be over- and underestimated by contrast-enhanced $\mathrm{MRI}^{[38]}$. Combined PET/MR imaging in neurooncology might evolve as a valuable tool for more exact delineation of the tumor extent before surgery or radiation therapy, for selection of appropriate biopsy sites, for follow-up of patients as a modality allowing differentiation of tumor recurrence and radiation necrosis, and possibly also for early therapy response assessment. Using PET/MR including functional MR sequences (e.g., MR spectroscopy) may help in preoperative grading of tumors and guiding of therapeutic decisions.

FDG-PET has proven value in neurologic applications, such as the detection of hypometabolic cortical patterns 


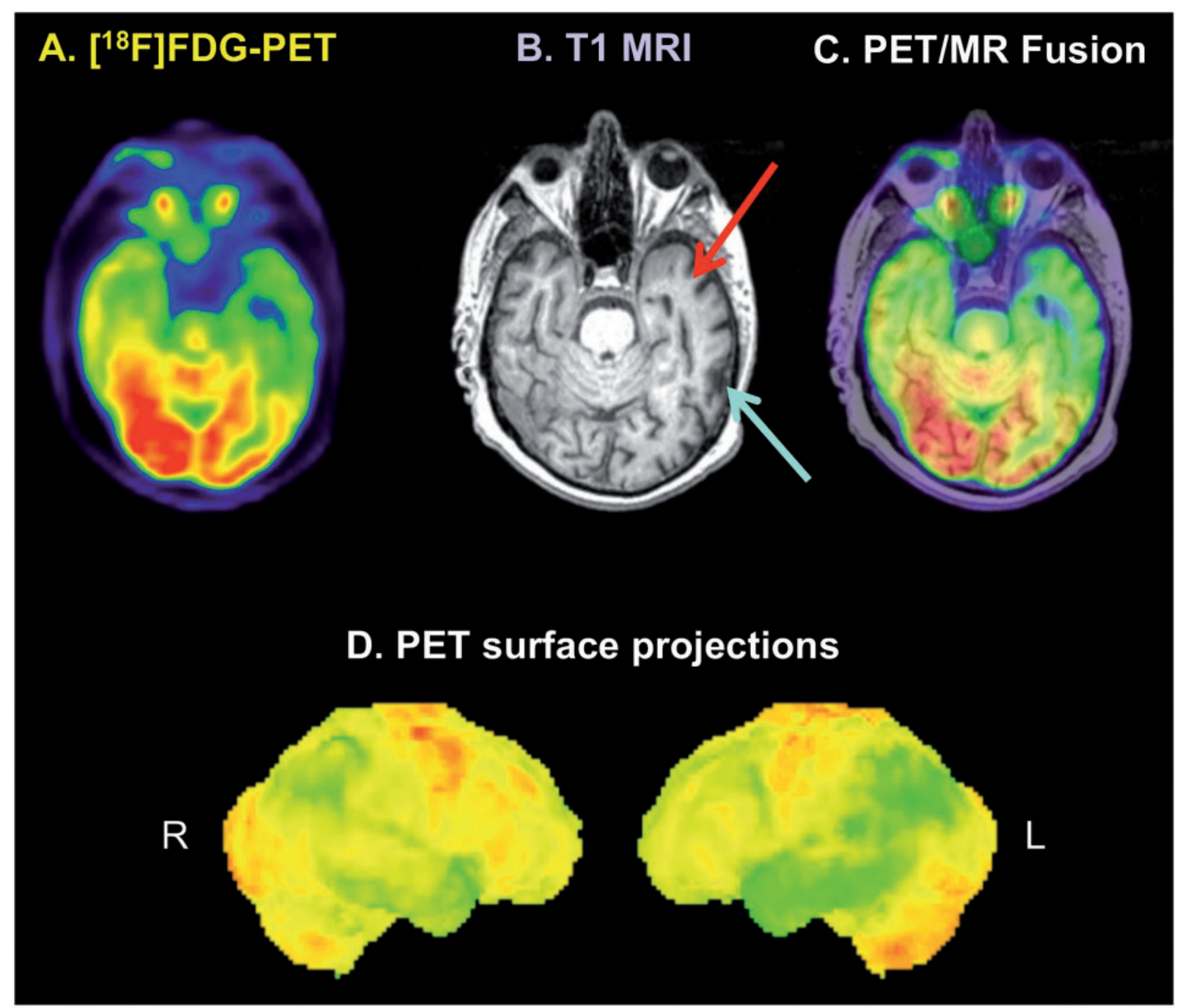

Figure 9 FDG-PET/MR in a patient with cognitive impairment and suspicion of a neurodegenerative disease. Cortical glucose hypometabolism is observed in the parietal and temporal lobes bilaterally, more pronounced in the left hemisphere (D), overall consistent with Alzheimer disease. Transaxial PET images (A) show most pronounced hypometabolism in the left temporal lobe, whereas T1-weighted MR images (B) and PET/MR fusion images (C) indicate that the decreased FDG uptake in the dorsal part of the left temporal lobe is due to cortical atrophy (blue arrow), whereas true glucose hypometabolism is present in the rostral parts of the temporal lobe without signs of cortical atrophy (red arrow). Image is courtesy of Alexander Drzezga.

for early diagnosis and differentiation of neurodegenerative diseases, and for the detection of hypometabolic epileptogenic foci. These applications benefit from the synergistic information delivered by PET and MRI, as well as both dementia ${ }^{[39]}$ and epilepsy ${ }^{[40]}$ studies (Fig. 9). A simultaneous PET/MR examination seems expedient for neurologic indications and offers a convenient one-stop-shop examination.

Truly simultaneous PET/MR brain scans are an exciting possibility for research applications, e.g., correlation of PET hypoxia markers (e.g., $\left[{ }^{18}\right.$ F]FMISO) with MR perfusion (DCE) and metabolic markers (MRS), validation of MR DWI and MR perfusion with PET perfusion measurements (e.g., $\left[{ }^{15} \mathrm{O}\right] \mathrm{H}_{2} \mathrm{O}$ ) in ischemic stroke, or evaluation of the effect of specific tasks on transmitter release and receptor binding by combining fMRI with dynamic PET measurements of receptor ligands ${ }^{[41]}$.

\section{Cardiology}

The possibility of quantifying tracer accumulation makes PET an ideal tool for cardiac imaging applications, because myocardial blood flow and coronary flow reserve can be quantitatively assessed by tracer kinetic modeling, e.g., using ${ }^{13} \mathrm{~N}$-labeled ammonia or ${ }^{15} \mathrm{O}$-labeled water. PET has emerged as the most robust noninvasive clinical tool for the assessment of myocardial viability and perfusion in patients with coronary artery disease (CAD) ${ }^{[42]}$. Its reported sensitivity and specificity for diagnosing obstructive CAD is about $90 \%^{[43]}$. MRI also offers a 


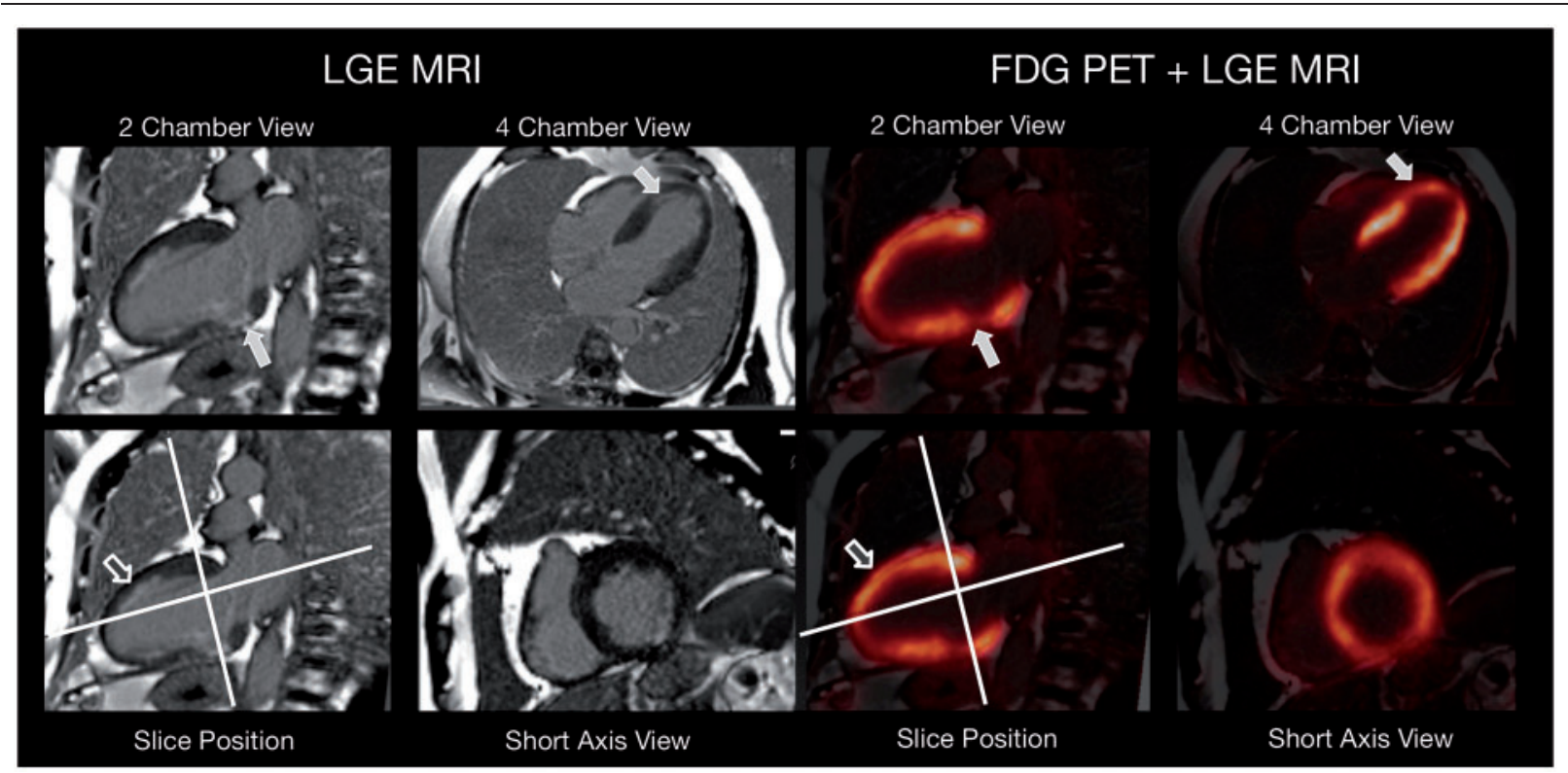

Figure 10 A 57-year-old man with cardiac 3-vessel disease and reduced left-ventricular function (ejection fraction 40\%). PET/MR was performed two months after anterior infarction and stenting of left anterior descending artery and first ramus posterolateralis to delineate myocardial viability. Late gadolinium enhancement (LGE) images revealed a nontransmural uptake pattern in the apex and the inferolateral wall with matching, modestly reduced, FDG uptake (filled arrows). However, note the nontransmural late gadolinium enhancement in the anterior wall (open arrow) with almost normal FDG uptake pointing to the synergetic effects of PET and MRI. Image is courtesy of Stephan G. Nekolla.

variety of cardiac imaging possibilities, including the assessment of cardiac structure and anatomy, ventricular function (e.g., volumes, ejection fraction, wall thickening and wall mass), and, in conjunction with the application of Gd-based contrast agent, assessment of myocardial perfusion and detection of postischemic myocardial scarring. Thus, in cardiac PET/MR, the MR component can deliver important information for the assessment of $\mathrm{CAD}$, complementing the information acquired by PET (Fig. 10).

PET using a combination of FDG and perfusion tracers offers the possibility of detecting hibernating myocardium, reflecting viable but jeopardized myocardium in patients with CAD. Hibernating myocardium is characterized by a mismatch of reduced perfusion but sustained or even increased glucose metabolism. The delayed enhancement assessed by MRI can reflect acute myocardial infarction or myocardial scarring caused by old infarctions, but not hibernating myocardium. Although there are indications that there is an association between PET mismatch and clinical outcome, prospective clinical trials will have to show whether PET and MRI can deliver data with complementary clinical information regarding myocardial viability ${ }^{[42]}$.

In cardiac studies, truly simultaneous acquisition of PET and MR could prove useful for minimization of misalignment between the two modalities, which is crucial in cardiac imaging to minimize artifacts in the PET data set ${ }^{[44]}$. During PET acquisition, repeated MR measurements for generation of attenuation maps and PET motion correction could be performed without additional radiation exposure for the patient. Simultaneous PET/ MR imaging could also offer MR-based cardiac and respiratory gating of the PET data without the need for external sensors. Furthermore, simultaneous rest/stress PET/ MR perfusion scans offer the possibility of cross-validating MR and PET perfusion measurements.

\section{Inflammation}

Infectious diseases of the bone, peripheral osteomyelitis as well as spondylitis and spondylodiscitis, can be difficult to diagnose. FDG accumulates in inflammatory processes due to uptake in activated lymphocytes, neutrophils, and activated macrophages, and FDG-PET as well as MRI are valuable clinical tools in the assessment of osteomyelitis ${ }^{[45]}$. High sensitivity and specificity in the diagnosis of chronic osteomyelitis have been reported for both modalities; FDG-PET has even outperformed MRI in some studies ${ }^{[46,47]}$. In diabetic patients with suspicion of osteomyelitis of the foot, MRI showed higher sensitivity, whereas PET had a higher specificity $^{[48]}$. MRI has special advantages differentiating soft tissue and bone infection, however its specificity can be impaired regarding osteomyelitis of the foot due to side findings of neuropathic arthropathy often present in diabetic patients (including, e.g., bony destruction and marrow edema), which are also morphologic signs of osteomyelitis on MR images ${ }^{[49]}$. Thus, the combination of PET and MRI might provide complementary information in these patients, improving sensitivity and 
specificity. Hybrid PET/MR might also be beneficial for imaging of spondylodiscitis as MRI offers a higher spatial resolution than PET, allowing the differentiation of involvement of vertebrae and vertebral discs. The use of MRI in treatment response evaluation of spinal infections is less clear $^{[49]}$, whereas FDG-PET could be potentially useful in the evaluation of treatment response ${ }^{[50]}$. Overall, PET/MR might prove to be a useful clinical tool for the diagnosis and response evaluation of bone infections, however no clinical data are presently available.

PET and MRI also seem to be a promising combination in the evaluation of vasculitis, e.g., giant cell arteritis or Takayasu arteritis. FDG-PET shows high sensitivity of about $80-90 \%$ for vasculitis of the large arteries ${ }^{[51,52]}$. Concerning smaller vessels, e.g., the superficial cranial arteries, MRI seems more promising as it has the ability to visualize the inflamed wall of smaller arteries (e.g., thickening) and Gd-contrast enhancement of the vessel wall, whereas due to its limited spatial resolution, the sensitivity of PET is impaired with respect to inflammation of smaller vessels. Furthermore, FDG uptake has been shown to correlate with the risk of later aneurysm formation, making it a prognostic marker, whereas aneurysm size and morphology can be visualized with MRI, adding additional value in the diagnosis and follow-up in patients with vasculitis ${ }^{[45]}$.

FDG-PET is also a suitable modality for the diagnosis of vascular graft infection ${ }^{[53]}$ and for the detection of infectious foci in patients with bacteremia ${ }^{[54]}$, however, the role of MRI (and PET/MR) in these scenarios remains to be established.

The conventional imaging modalities used in the diagnosis of inflammatory bowel disease are ultrasonography, $\mathrm{CT}$ and MRI. The latter provides the highest sensitivity of the 3 , especially for detecting signs of intestinal and mesenteric inflammation ${ }^{[49]}$. Available data about the use of FDG-PET for the evaluation of inflammatory bowel disease, albeit limited, suggest a similar sensitivity to MRI and leukocyte scintigraphy. Nonetheless, the potential benefits of combined PET/MR in the diagnosis and follow-up of patients with inflammatory bowel disease remain to be evaluated, and prospective clinical studies are warranted ${ }^{[45]}$.

\section{Conclusion}

The development of PET/CT successfully introduced hybrid imaging to clinical routine and it has been widely accepted in clinical imaging. The recent introduction of PET/MR offers new opportunities for research as well as clinical applications. The possibility of truly simultaneous acquisition of PET and MR data is probably most relevant for neurologic research applications. Furthermore, it offers new opportunities for cross-validation of the two methods, e.g., regarding quantitative myocardial blood flow assessment in cardiology. In oncology,
PET/MR holds promise to improve the accuracy and convenience of clinical staging and therapy response assessment, especially in indications and body regions where MR is known to be superior to CT, e.g., liver tumors and liver metastases, breast and prostate cancer, soft tissue sarcomas, neuroendocrine tumors, and neurooncology. However, only limited data are available on the clinical performance of the new hybrid imaging technology, and its clinical value remains to be evaluated in studies. Further clinical data are awaited eagerly.

In oncology, PET/CT has mostly replaced stand-alone PET. Rather than being repeated with PET/MR and PET/CT, the two hybrid imaging modalities will likely coexist, each of them offering specific advantages as well as shortcomings. Overall, the appropriate modality will have to be chosen individually for each patient depending on the indication.

The new technology also comes with challenges. Acquisition and maintenance costs of integrated wholebody PET/MR scanners are higher than those of PET/ CT. Also examination times tend to be longer, leading to presumably lower patient throughput, and image interpretation and reporting is more complex and time consuming. Therefore, the cost-efficiency of PET/MR needs to be evaluated.

\section{Acknowledgments}

The Department of Nuclear Medicine, Technische Universität München has established a research cooperation contract with Siemens Healthcare AG. M.S. has been invited to present lectures on PET/MR by Siemens Healthcare AG. This work has been supported by the Deutsche Forschungsgemeinschaft, Großgeräteinitiative, who funded the PET/MR scanner at the Technische Universität München, and the SFB824. This work was supported by a grant to S.F. by the Graduate School of Information Science in Health (GSISH) and the TUM Graduate School. We thank Gaspar Delso for providing the PET/MR system designs shown in Figs. 1-3. We thank Alexander Drzezga for the neurology PET/MR figure and Stephan G. Nekolla for the cardiac PET/MR images.

\section{References}

[1] Beyer T, Townsend DW, Brun T, et al. A combined PET/CT scanner for clinical oncology. J Nucl Med 2000; 41: 1369-1379. PMid:10945530.

[2] Czernin J, Allen-Auerbach M, Schelbert HR. Improvements in cancer staging with PET/CT: literature-based evidence as of September 2006. J Nucl Med 2007; 48(Suppl 1): 78S-88S. PMid:17204723.

[3] Wechalekar K, Sharma B, Cook G. PET/CT in oncology-a major advance. Clin Radiol 2005; 60: 1143-1155. doi:10.1016/ j.crad.2005.05.018. PMid:16223611.

[4] Picchio M, Mansueto M, Crivellaro C, et al. PET/CT and contrast enhanced CT in single vs. two separate sessions: a cost analysis study. Q J Nucl Med Mol Imaging 2012; 56: 309-316. PMid:22510702. 
[5] Townsend DW. Combined positron emission tomography-computed tomography: the historical perspective. Semin Ultrasound CT MR 2008; 29: 232-235. doi:10.1053/j.sult.2008.05.006. PMid: 18795489.

[6] Delso G, Ziegler S. PET/MRI system design. Eur J Nucl Med Mol Imaging 2009; 36(Suppl 1): S86-S92. doi:10.1007/s00259008-1008-6. PMid:19104809.

[7] von Schulthess GK, Burger C. Integrating imaging modalities: what makes sense from a workflow perspective? Eur J Nucl Med Mol Imaging 2010; 37: 980-990. doi:10.1007/s00259-0091378-4. PMid:20140613.

[8] Shao Y, Cherry SR, Farahani K, et al. Simultaneous PET and MR imaging. Phys Med Biol 1997; 42: 1965-1970. doi:10.1088/00319155/42/10/010. PMid:9364592.

[9] Pichler BJ, Judenhofer MS, Catana C, et al. Performance test of an LSO-APD detector in a 7-T MRI scanner for simultaneous PET/MRI. J Nucl Med 2006; 47: 639-647. PMid:16595498.

[10] Delso G, Fürst S, Jakoby B, et al. Performance measurements of the Siemens mMR integrated whole-body PET/MR scanner. J Nucl Med 2011; 52: 1914-1922. doi:10.2967/ jnumed.111.092726. PMid:22080447.

[11] Drzezga A, Souvatzoglou M, Eiber M, et al. First clinical experience with integrated whole-body PET/MR. Comparison to PET/ CT in patients with oncologic diagnoses. J Nucl Med 2012; 53: 845-855. doi:10.2967/jnumed.111.098608. PMid:22534830.

[12] Kinahan PE, Hasegawa BH, Beyer T. X-ray-based attenuation correction for positron emission tomography/computed tomography scanners. Semin Nucl Med 2003; 33: 166-179. PMid: 12931319.

[13] Hofmann M, Pichler B, Scholkopf B, Beyer T. Towards quantitative PET/MRI: a review of MR-based attenuation correction techniques. Eur J Nucl Med Mol Imaging 2009; 36(Suppl 1): S93-S104. doi:10.1007/s00259-008-1007-7. PMid:19104810.

[14] Martinez-Moller A, Souvatzoglou M, Delso G, et al. Tissue classification as a potential approach for attenuation correction in whole-body PET/MRI: evaluation with PET/CT data. J Nucl Med 2009; 50: 520-526. doi:10.2967/jnumed.108.054726. PMid:19289430.

[15] Martinez-Moeller A, Souvatzoglou M, Navab N, Schwaiger M, Nekolla SG. MR-based attenuation correction for whole-body MR/PET. J Nucl Med 2008; 49(Suppl 1): 65.

[16] Berker Y, Franke J, Salomon A, et al. MRI-based attenuation correction for hybrid PET/MRI systems: a 4-class tissue segmentation technique using a combined ultrashort-echo-time/Dixon MRI sequence. J Nucl Med 2012; 53: 796-804. doi:10.2967/ jnumed.111.092577. PMid:22505568.

[17] Townsend DW, Carney JP, Yap JT, Hall NC. PET/CT today and tomorrow. J Nucl Med 2004; 45(Suppl 1): 4S-14S. PMid: 14736831.

[18] Eiber M, Martinez-Moller A, Souvatzoglou M, et al. Value of a Dixon-based MR/PET attenuation correction sequence for the localization and evaluation of PET-positive lesions. Eur J Nucl Med Mol Imaging 2011; 38: 1691-1701. doi:10.1007/s00259011-1842-9. PMid:21688050.

[19] Brix G, Nekolla EA, Nosske D, Griebel J. Risks and safety aspects related to PET/MR examinations. Eur J Nucl Med Mol Imaging 2009; 36(Suppl 1): S131-S138. doi:10.1007/s00259008-0937-4. PMid:19104806.

[20] International Commission on Non-Ionizing Radiation Protection. Medical magnetic resonance (MR) procedures: protection of patients. Health Phys 2004; 87: 197-216. doi:10.1097/ 00004032-200408000-00008. PMid:15257220.

[21] Thomsen HS. Recent hot topics in contrast media. Eur Radiol 2011; 21: 492-495. doi:10.1007/s00330-010-2026-x. PMid: 21136062.

[22] Hasebroock KM, Serkova NJ. Toxicity of MRI and CT contrast agents. Expert Opin Drug Metab Toxicol 2009; 5: 403-416. doi:10.1517/17425250902873796. PMid:19368492.
[23] Brooks RA. A quantitative theory of the Hounsfield unit and its application to dual energy scanning. J Comput Assist Tomogr 1977; 1: 487-493. doi:10.1097/00004728-197710000-00016. PMid:615229.

[24] Wehrl HF, Judenhofer MS, Wiehr S, Pichler BJ. Pre-clinical PET/ MR: technological advances and new perspectives in biomedical research. Eur J Nucl Med Mol Imaging 2009; 36(Suppl 1): S56-S68. doi:10.1007/s00259-009-1078-0. PMid:19194703.

[25] Gaertner FC, Souvatzoglou M, Brix G, Beer AJ. Imaging of hypoxia using PET and MRI. Curr Pharm Biotechnol 2012; 13: 552-570. doi:10.2174/138920112799436267. PMid:22214501.

[26] Antoch G, Bockisch A. Combined PET/MRI: a new dimension in whole-body oncology imaging? Eur J Nucl Med Mol Imaging 2009; 36(Suppl 1): S113-S120. doi:10.1007/s00259-008-0951-6. PMid:19104802.

[27] Van Goethem M, Tjalma W, Schelfout K, Verslegers I, Biltjes I, Parizel P. Magnetic resonance imaging in breast cancer. Eur J Surg Oncol 2006; 32: 901-910. doi:10.1016/j.ejso.2006.06.009. PMid:16920327.

[28] Heusner TA, Kuemmel S, Umutlu L, et al. Breast cancer staging in a single session: whole-body PET/CT mammography. J Nucl Med 2008; 49: 1215-1222. doi:10.2967/jnumed.108.052050. PMid:18632831

[29] Souvatzoglou M, Gaertner FC, Schwarzenboeck S, Beer AJ, Schwaiger M, Krause BJ. PET/CT for the diagnosis, staging \& restaging of prostate cancer. Imaging Med 2011; 3: 571-585. doi:10.2217/iim.11.48.

[30] Beer AJ, Eiber M, Souvatzoglou M, Schwaiger M, Krause BJ. Radionuclide and hybrid imaging of recurrent prostate cancer. Lancet Oncol 2011; 12: 181-191. doi:10.1016/S14702045(10)70103-0. PMid:20599424.

[31] Collins CD. PET/CT in oncology: for which tumours is it the reference standard? Cancer Imaging 2007; 7(A): S77-S87.

[32] $\mathrm{Wu} \mathrm{LM}, \mathrm{Xu} \mathrm{JR}, \mathrm{Gu} \mathrm{HY}$, et al. Preoperative mediastinal and hilar nodal staging with diffusion-weighted magnetic resonance imaging and fluorodeoxyglucose positron emission tomography/computed tomography in patients with non-small-cell lung cancer: which is better? J Surg Res 2012; 178: 304-314. doi:10.1016/ j.jss.2012.03.074. PMid:22541065.

[33] Pauls S, Schmidt SA, Juchems MS, et al. Diffusion-weighted MR imaging in comparison to integrated $\left[{ }^{18} \mathrm{~F}\right]-\mathrm{FDG}$ PET/CT for $\mathrm{N}$ staging in patients with lung cancer. Eur J Radiol 2012; 81: 178-182. doi:10.1016/j.ejrad.2010.09.001. PMid:20932700.

[34] Saksena MA, Saokar A, Harisinghani MG. Lymphotropic nanoparticle enhanced MR imaging (LNMRI) technique for lymph node imaging. Eur J Radiol 2006; 58: 367-374. doi:10.1016/ j.ejrad.2005.12.041. PMid:16472955.

[35] Schmidt GP, Schoenberg SO, Schmid R, et al. Screening for bone metastases: whole-body MRI using a 32-channel system versus dual-modality PET-CT. Eur Radiol 2007; 17: 939-949. doi:10.1007/s00330-006-0361-8. PMid:16951929.

[36] Coenegrachts K, De Geeter F, ter Beek L, et al. Comparison of MRI (including SS SE-EPI and SPIO-enhanced MRI) and FDGPET/CT for the detection of colorectal liver metastases. Eur Radiol 2009; 19: 370-379. doi:10.1007/s00330-008-1163-y. PMid:18795299.

[37] Dikaios N, Izquierdo-Garcia D, Graves MJ, Mani V, Fayad ZA, Fryer TD. MRI-based motion correction of thoracic PET: initial comparison of acquisition protocols and correction strategies suitable for simultaneous PET/MRI systems. Eur Radiol 2012; 22: 439-446. doi:10.1007/s00330-011-2274-4. PMid: 21938440

[38] Klasner BD, Krause BJ, Beer AJ, Drzezga A. PET imaging of gliomas using novel tracers: a sleeping beauty waiting to be kissed. Expert Rev Anticancer Ther 2010; 10: 609-613. doi:10.1586/ era.10.37. PMid:20469990.

[39] Herholz K. PET studies in dementia. Ann Nucl Med 2003; 17: 79-89. doi:10.1007/BF02988444. PMid:12790355. 
[40] Koepp MJ, Woermann FG. Imaging structure and function in refractory focal epilepsy. Lancet Neurol 2005; 4: 42-53. doi:10.1016/S1474-4422(04)00965-2. PMid:15620856.

[41] Heiss WD. The potential of PET/MR for brain imaging. Eur J Nucl Med Mol Imaging 2009; 36(Suppl 1): S105-S112. doi:10.1007/s00259-008-0962-3. PMid:19104801.

[42] Nekolla SG, Martinez-Moeller A, Saraste A. PET and MRI in cardiac imaging: from validation studies to integrated applications. Eur J Nucl Med Mol Imaging 2009; 36(Suppl 1): S121-S130. doi:10.1007/s00259-008-0980-1. PMid:19104798.

[43] Klocke FJ, Baird MG, Lorell BH, et al. ACC/AHA/ASNC guidelines for the clinical use of cardiac radionuclide imaging-executive summary: a report of the American College of Cardiology/ American Heart Association Task Force on Practice Guidelines (ACC/AHA/ASNC Committee to Revise the 1995 Guidelines for the Clinical Use of Cardiac Radionuclide Imaging). J Am Coll Cardiol 2003; 42: 1318-1333. doi:10.1016/j.jacc.2003.08.011. PMid: 14522503.

[44] Martinez-Moller A, Souvatzoglou M, Navab N, Schwaiger M, Nekolla SG. Artifacts from misaligned CT in cardiac perfusion PET/CT studies: frequency, effects, and potential solutions. J Nucl Med 2007; 48: 188-193. PMid:17268013.

[45] Gotthardt M, Bleeker-Rovers CP, Boerman OC, Oyen WJ. Imaging of inflammation by PET, conventional scintigraphy, and other imaging techniques. J Nucl Med 2010; 51: 1937-1949. doi:10.2967/jnumed.110.076232. PMid:21078798.

[46] Termaat MF, Raijmakers PG, Scholten HJ, Bakker FC, Patka P, Haarman HJ. The accuracy of diagnostic imaging for the assessment of chronic osteomyelitis: a systematic review and meta-analysis. J Bone Joint Surg Am 2005; 87: 2464-2471. doi:10.2106/ JBJS.D.02691. PMid:16264122.

[47] Gratz S, Dorner J, Fischer U, et al. 18F-FDG hybrid PET in patients with suspected spondylitis. Eur J Nucl Med Mol Imaging 2002; 29: 516-524. PMid:11914890.
[48] Nawaz A, Torigian DA, Siegelman ES, Basu S, Chryssikos T, Alavi A. Diagnostic performance of FDG-PET, MRI, and plain film radiography (PFR) for the diagnosis of osteomyelitis in the diabetic foot. Mol Imaging Biol 2010; 12: 335-342. doi:10.1007/ s11307-009-0268-2. PMid:19816744.

[49] Glaudemans AW, Quintero AM, Signore A. PET/MRI in infectious and inflammatory diseases: will it be a useful improvement? Eur J Nucl Med Mol Imaging 2012; 39: 745-749. doi:10.1007/ s00259-012-2060-9. PMid:22297458.

[50] Treglia G, Focacci C, Caldarella C, et al. The role of nuclear medicine in the diagnosis of spondylodiscitis. Eur Rev Med Pharmacol Sci 2012; 16(Suppl 2): 20-25. PMid:22655480.

[51] Blockmans D, de Ceuninck L, Vanderschueren S, Knockaert D, Mortelmans L, Bobbaers H. Repetitive ${ }^{18} \mathrm{~F}$-fluorodeoxyglucose positron emission tomography in giant cell arteritis: a prospective study of 35 patients. Arthritis Rheum 2006; 55: 131-137. doi:10.1002/art.21699. PMid:16463425.

[52] Hautzel H, Sander O, Heinzel A, Schneider M, Muller HW. Assessment of large-vessel involvement in giant cell arteritis with ${ }^{18}$ F-FDG PET: introducing an ROC-analysis-based cutoff ratio. J Nucl Med 2008; 49: 1107-1113. doi:10.2967/ jnumed.108.051920. PMid:18552151.

[53] Spacek M, Belohlavek O, Votrubova J, Sebesta P, Stadler P. Diagnostics of "non-acute" vascular prosthesis infection using ${ }^{18}$ F-FDG PET/CT: our experience with 96 prostheses. Eur J Nucl Med Mol Imaging 2009; 36: 850-858. doi:10.1007/ s00259-008-1002-z. PMid:19107480.

[54] Bleeker-Rovers CP, Vos FJ, Corstens FH, Oyen WJ. Imaging of infectious diseases using $\left[{ }^{18} \mathrm{~F}\right]$ fluorodeoxyglucose PET. Q J Nucl Med Mol Imaging 2008; 52: 17-29. PMid:17657204.

[55] Martinez-Moller A, Eiber M, Nekolla SG, et al. Workflow and scan protocol considerations for integrated whole-body PET/MRI in oncology. J Nucl Med 2012; 3: 1415-1426. 